\title{
Post-Eruptive Flare Energy Release as Detected on AU Mic by EUVE
}

\author{
M. M. KATSOVA, ${ }^{1}$ J. J. DRAKE, ${ }^{2}$ AND M. A. LIVSHITS $^{3}$
}

\author{
${ }^{1}$ Sternberg State Astronomical Institute, Moscow State University, 119899 Moscow, Russia \\ ${ }^{2}$ Center for EUV Astrophysics, 2150 Kittredge Street, \\ University of California, Berkeley CA 94720-5030, USA \\ ${ }^{3}$ Institute of Terrestrial Magnetism, Ionosphere and Radio Wave Propagation, Russian \\ Academy of Sciences, 142092 Troitsk, Moscow Region, Russia
}

The long-duration emission arising after the impulsive rise and decay in a flaring event observed by the Extreme Ultraviolet Explorer on the red dwarf star AU Mic is discussed. The decay of the intensity in the Deep Survey 65-190 $\AA$ band and in the Fe XVIII line during this prolonged event is 10 times slower than the time of radiative cooling of coronal loops with the typical for the flare plasma density. The temporal behavior of the emission measure is determined for both the 65-190 $\AA$ band and the Fe XVIII line fluxes. The total energy emitted in the 1-2000 $\AA$ region over nearly $12 \mathrm{hrs}$ is $3 \cdot 10^{35}$ ergs. We first point out some difficulties with earlier explanations proposed for this event; we then propose the following physical model: the source of the prolonged emission is a system of high coronal loops, the size of which is more than the active region scale, but less than the stellar radius. Such systems are observed in soft X-rays during large solar flares after coronal mass ejections. Some additional post-flare energy input into this high coronal loop system can be caused by reconnection in a vertical current sheet, and this post-eruptive energy release provides prolonged and intensive EUV emission.

Apparently, we are faced here with new kind of the surface activity on late-type stars which is intermediate between impulsive flares on red dwarfs and long-duration, powerful events the subgiants components of the RS CVn binaries.

\section{Observations}

AU Mic was observed by EUVE from 12:28 UT 14 July 1992 to 8:09 UT 18 July 1992. The Deep Survey (DS) data have been discussed in previous papers (Cully et al. 1994; Drake et al. 1994; Landini \& Monsignori Fossi 1994). We illustrate the DS light curve from Cully et al. in Figure 1. The description of the spectrometer data reduction will appear in a forthcoming paper (Brown et al. 1995); a very brief description is provided in Drake et al. (1994).

In this study, we represent the flare decay using an isothermal model with a time dependent temperature $T(t)$. The physical conditions were determined from the DS photometer data and the intensity of lines of Fe seen in the SW and MW spectrometers. The estimation of the temperature and its temporal behaviour was based on the behaviour of Fe lines from charge states XVIII, XXI, XXII, XXIII and XXIV. These lines indicate that, following the impulsive phase, the temperature decreases gradually from the value of $\sim 1 \cdot 10^{7} \mathrm{~K}$. The estimated $E M$, based on the DS and Fe XVIII data, and $T$ as a function of time are presented in Figure 2. The intensity of the Fe XVIII line emission decreases faster than the DS count rate independently from the chosen temporal behaviour of the temperature. The maximum EM of the flare decay estimated from both from the DS and spectral data is $1.6 \cdot 10^{53} \mathrm{~cm}^{-3}$ - similar to the values derived by Cully et al. (1994) from the DS photometer data.

Three possible explanations of this long duration EUV radiation are discussed:

(1) the flare X-ray loops; 


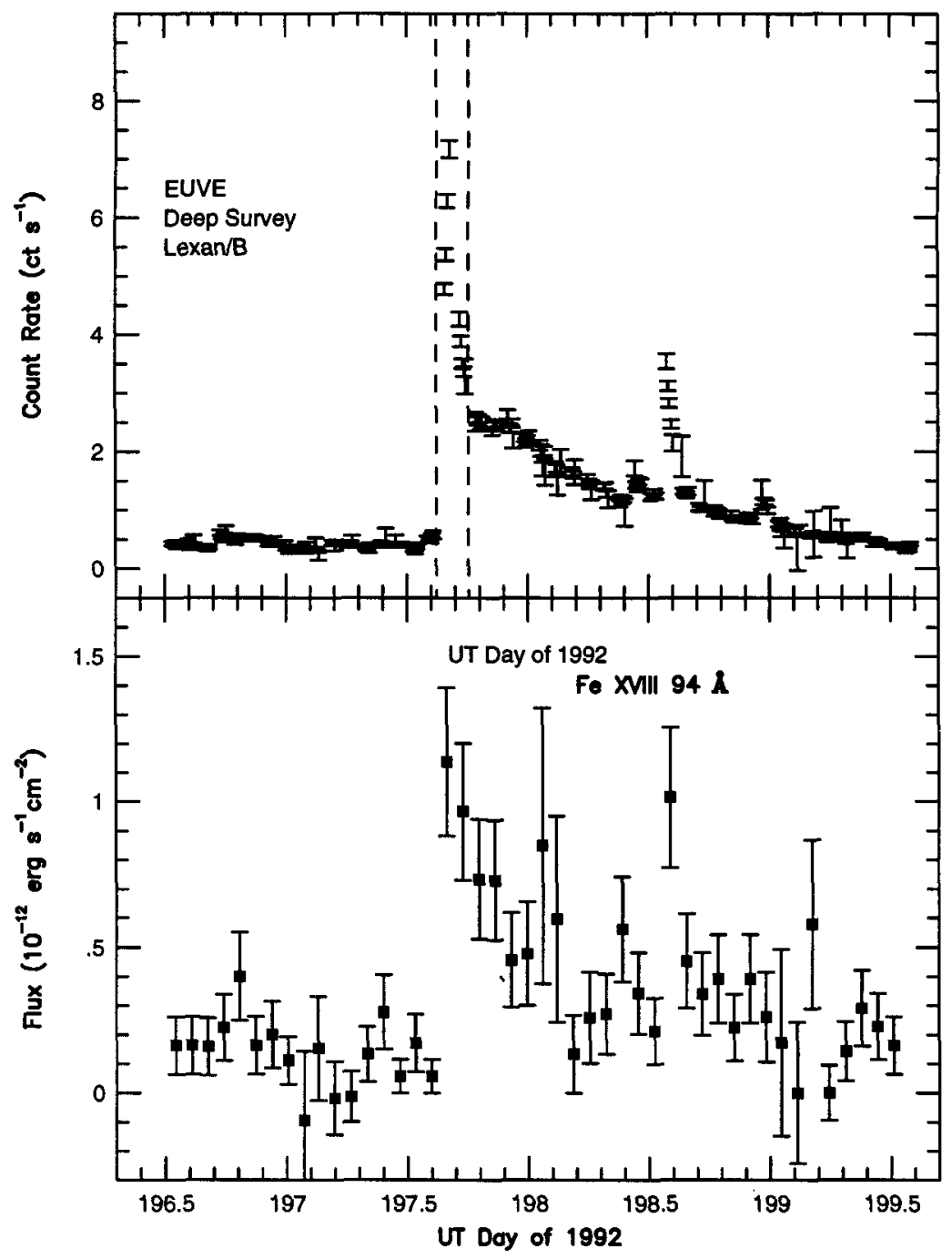

FIGURE 1. Temporal behaviour of the EUV emission observed from the July 14-17 1992 flare on AU Mic. Top panel: the 65-190 $\AA$ Deep Survey count rate (from Cully et al. 1994). Bottom panel: the Fe XVIII $93.9 \AA$ line (Drake et al. 1994).

(2) very high coronal loops;

(3) coronal mass ejection (CME), cf. Cully et al., 1994.

The soft X-ray emission of stellar flares does not usually exceed $2.3 t_{\text {rad }}$ or about of 1 hour; in these cases the soft X-ray intensity decay is caused by radiative cooling. The plasma radiative cooling time is given by

$$
t_{\text {rad }}=\frac{3 k T}{n L(T)}
$$




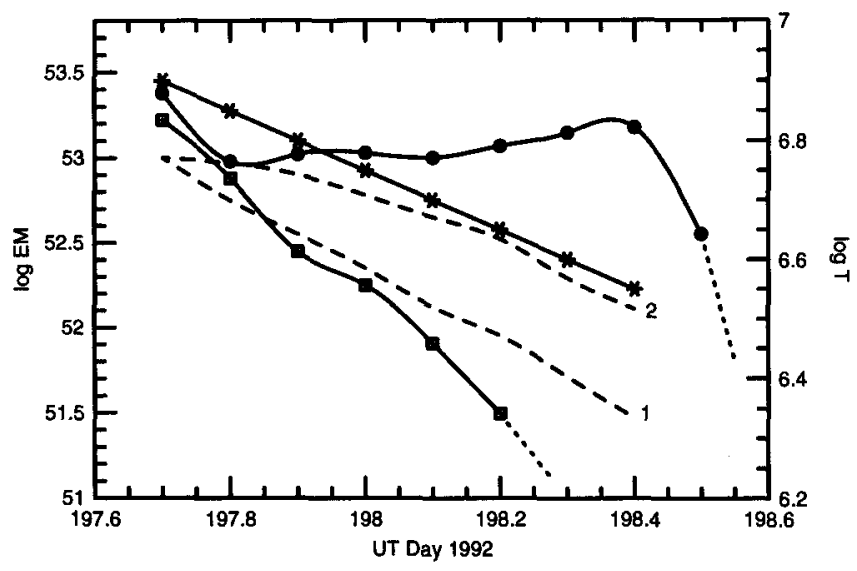

FIGURE 2. Emission measure vs. time calculated for the flare decay for the 65-190 $\AA$ DS data (solid dots) and the Fe XVIII line (hollow squares). The curve delineated by stars shows the chosen behaviour of the temperature as a function of time, $T(t)$. Theoretical calculations (dashed lines) are given for the case of $30 \%$ compensation of the radiative losses by additional heating and the initial density $n_{o} \sim 5 \cdot 10^{9} \mathrm{~cm}^{-3}$ : (1) for the chosen temporal temperature dependence $T(t) ;(2)$ for the isothermal case.

\begin{tabular}{lcccc}
\hline & $\begin{array}{c}\text { Volume } \\
\mathrm{cm}^{-3}\end{array}$ & $\begin{array}{c}\text { Density } \\
\mathrm{cm}^{-3}\end{array}$ & $\begin{array}{c}t_{\text {rad }} \\
\mathrm{s}\end{array}$ & $t_{\text {obs }} / t_{\text {rad }}$ \\
1 & $2 \cdot 10^{29}$ & $10^{12}$ & 140 & 320 \\
2 & $2 \cdot 10^{31}$ & $>10^{10}$ & 1700 & 26 \\
3 & $10^{35}$ & $1 \cdot 10^{9}$ & $>10^{5}$ & $<1$ \\
\hline
\end{tabular}

where $n^{2} L(T)$ is the radiative loss function (1-2000 $\AA$ ) in erg $\mathrm{cm}^{-3} \mathrm{~s}^{-1}$. We compare $t_{\text {rad }}$ for typical parameters of the three scenarios mentioned above in Table 1.

As seen in Table 1, the observed duration of the EUV emission decay is much longer than the radiative cooling time in the model (1) and (2). Indeed, the total duration of the decay of the DS $65-190 \AA$ band count rate and of the Fe XVIII line during the prolonged event on $\mathrm{AU} \mathrm{Mic}$ is 10 times longer than the radiative cooling time of coronal loops with typical flare densities.

The long-duration event emits as a whole

$$
W=\int n^{2} L(T) d t d V=\int E M_{D S} L(T) d t
$$

i.e., about of $W=3 \cdot 10^{35}$ ergs. This exceeds the energetics of typical flares on red dwarf stars, and even more so the energetics of solar flares. 


\section{How Does the Solar Analogy Help to Explain the Stellar Phenomenon?}

The similarity in the general appearance of the AU Mic event and large solar flares was pointed out by Drake et al. (1994), who noted that such an analogy would require substantial post-flare heating in order to compensate for radiative losses.

Several long-duration soft X-ray events were observed on the Sun during solar cycle XXII. The 1-8 $\AA$ radiation lasts from about 0.5 to 1 day. Different aspects of such solar events with soft X-ray emission lasting longer than 0.5 day are discussed by Cliver et al. (1986), Harrison (1986), Smith et al. (1994) and Akimov et al. (1993). Based on the combination of these results, Chertok (1993) have suggested that the post-eruptive energy release for such long duration solar flares can be explained within the framework of the theory of Martens (1988): a coronal mass ejection radially distends the magnetic loop force lines which then form a large-scale vertical current sheet. Subsequent plasma instabilities and reconnection in this vertical current sheet are able, apparently, to accelerate the particles up to very high energies like $10^{10} \mathrm{eV}$. Note that this phenomenon is observed as a system of cool $\mathrm{H}_{\alpha}$ post-flare loops lying lower than the soft $\mathrm{X}$-ray loops. Such systems were observed during powerful solar flares such as those of September 29 1989 , and June 151991 within 12 hrs of the onset of the impulsive phase. If additional heating does compensate for the radiative losses, then the hot dense loops can exist as long as this heating lasts. These solar observations are now considered as some of the main evidence for this post-eruptive phase of solar flares.

The solar results also support the point of view that a CME alone is not able to provide the observed fluxes of EUV and soft X-ray emission over many hours. Therefore, despite the fact that the energetics of CME and the type of event considered above are rather similar, the third model (CME) is probably not the best explanation of long-duration EUV emission both on the Sun and on red dwarfs. In particular, the interpretation of the AU Mic event in terms of a CME alone by Cully et al. (1994) might be questioned, because the theoretical temporal behaviour of the density in the CME does not agree with optical data for solar CME's. A possible test of this model is the observation or not of the expected blue-shifted or red-shifted lines indicative of velocities of $\sim 1000 \mathrm{kms}^{-1}$ or so.

Thus, we have to explain the long timescale for the decay of the observed post-flare EUV flux from AU Mic. In principle, it could be due to flaring loops, the size of which does not exceed the typical scale of active regions (the Model 1 in Table 1). In such a case, emission should be observed not only in the EUV but also in soft X-rays such as was detected by EXOSAT and EINSTEIN. We note that there are no cases of such prolonged events observed with these satellites (Pallavicini et al., 1990). These dense $\left(n_{\varepsilon} \sim 10^{13}\right.$ $\mathrm{cm}^{-3}$ in the second pulse; Brown, 1994) flaring loops should cool down rapidly due to radiative losses in the radiative cooling timescale $t_{\text {rad }}$. In order for such an event to last for $100 t_{\text {rad }}$ or so, total compensation for the radiative losses over this period is required. Small-scale flaring processes are not able, in principle, to support additional heating with total energy $W=3 \cdot 10^{35}$ ergs in the $1-2000 \AA$ range. Therefore, there we are lead naturally to consider processes occurring on a larger scale (comparable to, or larger than the stellar radius). This, then, is the Model 2 in the Table).

Thus, the following model can be proposed for the explanation of these EUV observations:

If, as on the Sun, we consider the first pulse of the EUV light curve to be caused by an impulsive flare, so the gradual decay after this impulse can be presented as the radiation of the system of coronal loops which are forming after the CME. Figure 3 shows the 


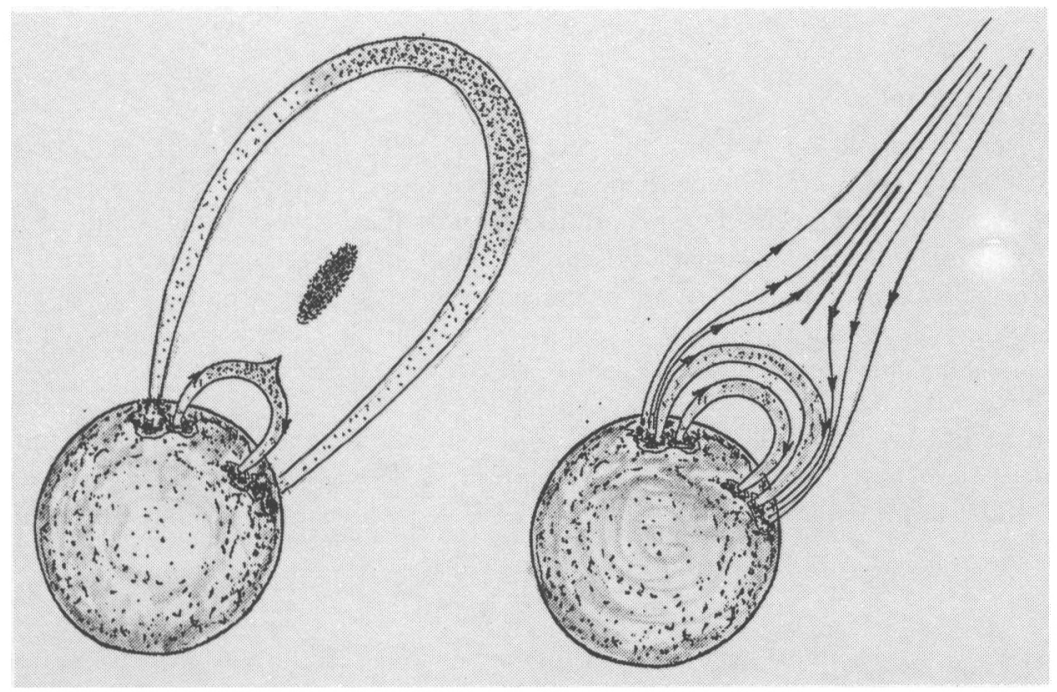

FIGURE 3. A schematic of the post-eruptive energy release. Left: CME as upper part of the highest loop; active prominence or plasmoid; hot coronal flare loop. Right: the phase after the coronal mass ejection (post-eruptive phase); magnetic force lines, vertical current sheet and long-lived hot, high coronal loops emitting in the soft X-ray and EUV range (see also Martens 1988).

CME as the upper part of an extended coronal loop and it is observed often in white light. The surge (active prominence) follows it, and the energy release in this casp (X-) point heats the plasma inside the X-ray coronal loop. The plasma motions extend the force lines of quasi-dipole magnetic field, forming the large-scale vertical current sheet (Martens et al. 1986), which make available additional heating of plasma in the coronal loops. This prolongs the life time of the coronal loops, which emit in the EUV spectral range. This process is similar to the solar case, but the energy input during the longduration EUV event should be $(2-10) \cdot 10^{30} \mathrm{ergs} \mathrm{s}^{-1}$ - a factor of 30 more than the relevant solar values.

We consider the balance of the energy in the EUV source without taking into account the detailed magnetohydrodynamic phenomena. If, for simplicity, we assume that the heating is proportional to the pressure, then we can write for $1 \mathrm{~cm}^{3}$ (or for all the volume which stays constant during this process):

where $E=3 n k T$ is inner thermal energy, the time independent value $\beta$ erg $\mathrm{cm}^{-3} \mathrm{~s}$ is the initial heating, $p_{o}$ is the initial pressure.

We have also derived the analytical solution of the equation of the energy balance when radiative losses are partially compensated by additional heating. Calculated values of emission measure for the isothermal case and the temperature dependence, $T(t)$, chosen above are illustrated in Figure 2 for the case of additional post-flare heating amounting to $30 \%$ of the radiative losses.

To explain the long duration of the post-flare EUV emission we need a large value of $t_{\text {rad }}$, which in turn implies a lower density during this phase as compared to the impulsive 
phase. Available spectral data and this theoretical consideration lead us to conclude that Model 2 in Table 1 seems to be acceptable.

\section{Conclusions}

The event observed on AU Mic on July 15-17, 1992 can be considered as a stellar analog of a typical solar flare with post-eruptive energy release. We argue that the long timescale for this event requires additional post-flare energy input. This energy is provided by reconnection in a vertical current sheet in a system of high coronal loops formed after a CME. Apparently, we are faced here with a new kind of surface activity on late-type stars which is intermediate between impulsive flares commonly seen on red dwarfs and long-duration, powerful events on RS CVn binaries.

MMK and MAL would like to thank the International Science Foundation for financial support of their participation in this Colloquium. JJD was supported by NASA grant AST91-15090 administered by the Center for EUV Astrophysics, University of California.

\section{REFERENCES}

AkIMov, V. V. ET AL. 1993, in Proc. 23rd International Cosmic Rays Conference, Calgary

Brown, A. 1994, in Cool Stars, Stellar Systems and the Sun, The 8th Cambridge Workshop, ed. J.-P. Caillaut, ASP Conf. Ser., 64, 23

Chertok, I. M. 1993, Astronomy Reports of the Russian Academy of Sciences, 37(1), 87

Cliver, E. W., Dennis, B. R., \& Kiplinger, A. L., et al., 1986, ApJ, 305, 920

Cully, S. L., Fisher, G., Abbott, M. J., \& Siegmund, O. H. W., 1994, ApJ, 435, 449

Drake, J. J., Brown, A., Bowyer, S., Jelinsky, P., Malina, R. F., \& WU, X. Y. 1994, in Cool Stars, Stellar Systems and the Sun. The 8th Cambridge Workshop, ed. J.-P. Caillaut, ASP Conf. Ser., 64, 35

HARRISON, R. H. 1986, A\&A, 162, 283

Monsignori Fossi, B. C. \& LANDinI, M. 1994, A\&A, 284, 900

Martens, P. C. H. 1988, ApJ, 330, L131

Pallavicini, R., Tagliaferri, G., \& Stella, L. 1990, A\&A, 228, 403

Smith, K. L., SvestKa, Z., Strong, K. T., \& McCabe, M. K. 1994, Sol. Phys., 149, 363 